\title{
Modelos de ANÁlISE HIDRÁULICA DE REDES DE DISTRIBUIÇÃO DE ÁGUA CONSIDERANDO DEMANDA DIRIGIDA PELA PRESSÃO E VAZAMENTOS
}

\section{WATER DISTRIBUTION NETWORKS ANALYSIS CONSIDERING LEAKAGE AND PRESSURE DRIVEN DEMAND MODELING}

\begin{abstract}
RESUMO
As ferramentas para análise hidráulica são componentes importantes na avaliação do funcionamento das redes de distribuição de água para abastecimento. Existem diversos métodos que podem ser utilizados para essa análise, no entanto, os modelos que procuram resolver o sistema de equaçóes correspondente através do método Newton-Raphson ou por meio de linearizações sucessivas são os mais eficientes. Quatro formulações baseadas nestes esquemas são avaliadas neste trabalho. O objetivo deste trabalho é fazer uma comparação dos métodos Newton-Raphson, Teoria Linear, Híbrido e Gradiente para a análise de redes de distribuição de água em regime permanente, considerando a demanda dirigida pela pressão e os Vazamentos. Para tanto, foi utilizado um layout de rede frequentemente empregado na literatura dotado de válvulas. $\mathrm{O}$ método do Gradiente foi o que convergiu em um número menor de iterações para redes mais simples, o Método Híbrido foi o que mais se adaptou para sistemas mais complexos.
\end{abstract}

KLEBBER TEODOMIRO MARTINS FORMIGA

Engenheiro Civil -UFCG. Mestre em Engenharia Civil - UFCG. Doutor em Hidráulica e Saneamento EESC/USP. Professor Adjunto da Escola de Engenharia Civil UFG

\section{FAZAL HUSSAIN CHAUDHRY}

Engenheiro Civil - Punjab University. Mestre em Engenahria Hidráulica - Asian Institute Of Technology. Doutor em Engenharia Civil - Colorado State University. Professor Titular do Departamento de Hidráulica e Saneamento EESC/USP

\author{
Recebido: 30/04/07 Aceito: 08/04/08
}

PALAVRAS-CHAVE: Modelagem, redes de distribuição de água, vazamentos, demanda dirigida pela pressão.

\begin{abstract}
The hydraulic analysis tools are important in the performance evaluation of water distribution networks. Various methods are available for such analysis. However, the hydraulic models that solve the system of equations describing the flow problem through Newton-Raphson or through its successive linearizations are the most efficient. It is the purpose of this paper to compare Newton-Raphson, Linear Theory, Hybrid and Gradient methods for steady-state hydraulic network analysis, considering leakage and pressure driven demand modeling. The network layout with hydraulic components frequently used in the literature was employed for this analysis. The Gradient method was found to produce best results in terms of number of iterations for the more simple networks, whereas the Hybrid method was better for more complex networks.
\end{abstract}

KEYWORDS: Modeling, water distribution networks, leakage, pressure driven demand.

\section{INTRODUÇÃO}

A análise de redes de distribuição de água consiste na forma mais eficiente de previsão do comportamento do sistema - vazóes e perdas de carga nos trechos, pressóes e cargas hidráulicas nos nós - quando ocorrem modificações devido a incrustações, mudança da rugosidade em virtude do envelhecimento dos tubos, vazamentos e quebras, entre outras situações comuns às redes. $\mathrm{O}$ problema hidráulico correspondente pode ser posto como o de determinação das vazões nos di- versos trechos da rede, de maneira a satisfazer as condiçōes expressas através das equações da conservação de massa nos nós e de energia nos trechos da rede.

Como as equações do problema não são lineares, este só pode ser resolvido diretamente em apenas algumas situações especiais. No entanto, na maioria dos casos, é preciso empregar métodos iterativos assumindo uma solução aproximada a cada passo até que o problema tenha sido resolvido, tais métodos podem ser classificados da seguinte forma: a) gradiente local; b) Newton-Raphson; c) linearização; d) minimização numérica; e e) métodos híbridos.

Os métodos pertencentes às três primeiras classes utilizam ferramentas de resolução de sistemas de equações não lineares para achar a resposta do problema, enquanto que o quarto método transforma o problema na minimização de uma função não linear convexa, considerando restrições de igualdade e desigualdade lineares. Os métodos híbridos são resultados da união de dois ou mais métodos anteriores.

Os métodos Hardy Cross (MHC) baseiam-se no trabalho de Cross (1936) em que o sistema de equaçóes original 
é resolvido empregando o conceito de gradiente local. Embora sejam os mais fáceis de calcular manualmente, os MHC e suas derivaçôes podem apresentar um comportamento instável, não convergindo para redes com muitas malhas, sendo muito dependente da escolha dos anéis para se efetuar o balanço de energia. Além disso, o método perde eficiência numérica quando aplicado às redes de grande porte, pois apresenta uma taxa de convergência muito baixa (Lemieux, 1972). Outra desvantagem desta formulação está na dificuldade de se acrescentar dispositivos na rede como bombas e válvulas redutoras de pressão.

A segunda classe de métodos resolve o sistema de equações não lineares por meio do método Newton-Raphson - MNR (Shamir e Howard, 1968). Este método, embora mais eficiente que o MHC, apresenta deficiências inerentes à formulação que depende principalmente dos valores iniciais para que apresente uma convergência rápida. A falta desses valores pode ser crítica em sistemas mais complexos.

Os métodos de linearização das equações, com destaque para o Método da Teoria Linear - MTL (Wood e Charles, 1972; Isaacs e Mills, 1980), empregam aproximações lineares sucessivas da equação de perda de carga nos trechos, produzindo bons resultados. A principal vantagem deste método são suas propriedades de convergência, que diferentemente do $\mathrm{MNR}$, não requer boas soluçōes iniciais. No entanto a taxa de convergência das iteraçōes finais deste método é inferior ao MNR (Nielsen, 1989).

O emprego de técnicas de otimização na análise de redes é feito de modo a minimizar uma função objetivo não linear e convexa, sujeita a um conjunto de equações lineares de restrições que representam as equações de conservação de massa nos nós e energia nos trechos. A principal vantagem deste método está na formulação, uma vez que a convexidade da função objetivo, combinada com a linearidade de equaçóes garante a existência e a unicidade da solução. Infelizmente, a solução numérica para este problema requer a utilização de um método de otimização que possa ser eficiente até mesmo quando aplicado a redes de grande porte. Embora as técnicas de otimização não lineares estejam avançadas atualmente, estas ainda enfrentam problemas de dimensionalidade, ou seja, para redes de grande porte, a capacidade e o tempo de processamento necessário são muito elevados. Exemplos de métodos que utilizam otimização para a análise hidráulica de redes são os trabalhos de Collins, Cooper, Helgason et al (1978) e Gomes (2004) que empregam respectivamente o Convex Simplex Method e o Generalized Reduced Gradient (GRG2), na resolução deste problema.

Os métodos híbridos incorporam vantagens de dois, ou mais métodos acima citados de modo a reduzir as limitaçōes dos mesmos. O Método do Gradiente (Todini e Pilati, 1987) efetua a união de técnicas de minimização numérica com o método Newton-Raphson. Esta união resultou em um algoritmo muito eficiente e que implicou na sua utilização em quase todos os pacotes de simuladores hidráulicos atualmente disponíveis como: EPANET, WATERCAD, SARA, MIKENET, PIPENET. Outro método híbrido foi o proposto por Nielsen (1989) que uniu as melhores características do MNR e MTL, resultando em um método robusto, capaz de convergir, em um número muito menor de iteraçōes, quando comparado com os resultados obtidos com essas técnicas isoladas (Souza, 1994).

Embora a adoção destes modelos de análise tenha avançado nos últimos anos, eles ainda carecem de um melhor refinamento que possibilite simular de modo mais realista as condiçóes reais do sistema (Todini, 2003). Estes requisitos são ainda mais importantes em estudos mais específicos da rede, como análise de perdas por vazamentos e avaliação da confiabilidade do sistema. Para tanto, é necessário efetuar uma modificação dos métodos desenvolvidos até então. Esta é feita incorporando modelos de vazamentos e de demanda dirigida pela pressão (DDP) à formulação hidráulica tradicional (Formiga, 2005).

Considerando que o vazamento é consequência direta de defeitos na rede, pode-se caracterizá-lo baseado na equação de descarga em orifícios (Jowitt e Xu, 1990). Desta forma, para avaliar as perdas o método de análise hidráulica deve considerar que ocorrerão fugas de água na rede e que estas serão proporcionais à pressão. Alguns métodos de avaliar estas perdas foram propostos por Germanopoulos e Jowitt (1989), Martínez, Conejos e Vercher (1999) e Tucciarelli, Criminisi e Termini (1999).

A finalidade básica dos sistemas de distribuição de água é transportar água de um lugar para o outro, garantindo que esta chegue em quantidade e qualidade desejadas ao usuário final. Assim, ao disponibilizar água ao consumidor, é preciso que exista ainda uma sobra de energia que será usada para superar as perdas de carga dentro dos imóveis. Se a pressão não for adequada, a quantidade de água suprida será menor do que aquela requerida, mas, não será nula, havendo então um atendimento parcial das demandas. No entanto, se a pressão estiver abaixo de determinado valor, que depende de cada caso, não haverá suprimento de nenhuma demanda (Wagner, Shamir e Marks, 1988).

Desta forma, ao se tentar modelar este comportamento, deve-se levar em conta a diminuição das demandas atendidas, o que modifica em parte o cenário que se está simulando. A forma de se avaliar esse fenômeno é considerar a demanda também como uma variável, que teria o seu valor calculado em função da pressão nos nós, ou seja, tem-se a demanda dirigida pela pressão (DDP). Algumas formulaçôes de DDP foram propostas por Wagner, Shamir et al (1988), Reddy e Elango (1989) e Fujiwara e Ganesharajah (1993). Gupta e Bhave (1996) fizeram um comparativo de diferentes técnicas que calculam a demanda como dependente da pressão, concluindo que os métodos parabólicos foram os que apresentaram melhores resultados.

A introdução dos modelos de vazamento e demanda dirigida pela pressão (DDP), no algoritmo de análise hidráulica foi proposta teoricamente por Salgado, Rojo e Zepeda (1993) para o método gradiente. No entanto, a adoção direta deste provoca instabilidade no modelo (Ackley, Tanyimboh, Tahar et al, 2001), podendo inviabilizar a sua utilização em algumas situações. Para se contornar este problema, foram desenvolvidas alguma rotinas para o cálculo da DDP e dos vazamentos (Martínez, Conejos et al, 1999; Todini, 2003, Soares, Cheung, Reis et al, 2004), no entanto, nestes trabalhos, o cálculo dos vazamentos era efetuado fora do algoritmo de análise hidráulica, devendo os valores das demandas serem recorrentemente atualizados. Embora este procedimento possibilite a convergência dos resultados, a sua eficiência é limitada, visto que o número de iteraçōes necessário ao cálculo é elevado.

Este trabalho tem por finalidade efetuar a inclusão da DDP e dos vazamentos explicitamente na formulação dos métodos de análise hidráulica, verificando a eficiência e estabilidade numérica dos mesmos. 


\section{MÉTODOS DE ANÁLISE HIDRÀULICA DE REDES}

A seguir será feita uma explanação sobre a formulação dos métodos avaliados neste trabalho. No desenvolvimento do modelo de análise hidráulica será considerada a seguinte convenção: para um trecho i que conecte dois nós $\mathrm{j}_{\mathrm{i}, 1} \mathrm{e}$ $\mathrm{j}_{\mathrm{i}, 2}$ Seja uma rede qualquer composta por NN nós interiores, NR nós fontes (reservatórios ou tanques), e NT trechos que conectam os nós. A perda de carga entre dois nós, $\mathrm{j}_{\mathrm{j}, 1}$ e $\mathrm{j}_{\mathrm{j}, 2}$, será expressa por:

$\mathrm{h}_{\mathrm{ji}, 2}-\mathrm{h}_{\mathrm{ji}, 1}=\mathrm{K}_{\mathrm{i}} \mathrm{q}_{\mathrm{i}}^{\mathrm{a}}$

em que h é a carga hidráulica no nó j, Ki é o coeficiente de resistência da tubulação no trecho i; a é o expoente da fórmula de perda de carga.

\section{Método Newton-Raphson (MNR)}

O método Newton-Raphson (Shamir e Howard, 1968) para a resolução de sistemas de equações não lineares é desenvolvido a partir da seguinte equação:

$\mathrm{h}_{\mathrm{k}+1}=\mathrm{h}_{\mathrm{k}}+\Delta \mathrm{h}_{\mathrm{k}}$

em que h é o vetor de carga hidráulica nos nós da rede na iteração $k$, e $\Delta \mathrm{h}$ é o gradiente da função de balanço de massa nos nós, obtido a partir da matriz Jacobiana conforme procedimento demonstrado por Nielsen (1989).

Desenvolvendo este sistema (Souza, 1994) obtêm-se a equação matricial expressa por:

$\mathrm{h}_{\mathrm{k}}+1=\mathrm{h}_{\mathrm{h}}-\mathrm{a} 7 \mathrm{~J}_{\mathrm{k}} \mathrm{A}^{-1} \mathrm{Q}+$

$+\mathrm{A}^{\mathrm{T}} \mathrm{C}\left(\mathrm{Ah}_{\mathrm{k}}+\mathrm{A}_{\mathrm{r}} \mathrm{h}_{\mathrm{r}}\right)$.

em que $\mathbf{A}$ é a matriz de incidência da rede, a é o expoente da fórmula de perda de carga, $\mathbf{Q}$ é o vetor de demanda nos nós, $\mathbf{h}$ é a carga disponível nos reservatórios do sistema, e $\mathbf{J}_{\mathrm{k}}$ é a matriz Jacobiana expressa por :

$\mathrm{J}_{\mathrm{k}}=\mathrm{A}^{\mathrm{T}} \mathrm{CA}$

em que $\mathrm{C}$ é a matriz diagonal:

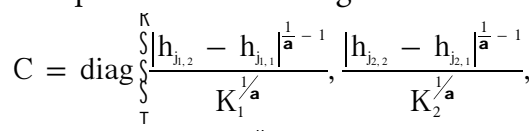

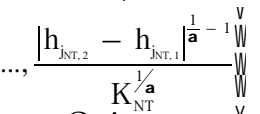

O sistema exp̀resso em (3) é resolvido sucessivamente até que a solução encontrada atenda a uma precisão pré-determinada, ou seja, a diferença das variáveis entre duas iteraçôes seja pequena.

\section{Método da Teoria Linear (MTL)}

O método da Teoria Linear (MTL) foi proposto inicialmente por Wood e Charles (1972) para formulação dos anéis, e modificado posteriormente por Isaacs e Mills (1980) para a formulação dos nós. Este método lineariza (1), resultando:

$\mathrm{h}_{\mathrm{ji}, 2 \mathrm{k}}-\mathrm{h}_{\mathrm{j}, \mathrm{i}_{\mathrm{lk}}}=\mathrm{K}_{\mathrm{i}} \mathrm{q}_{\mathrm{i}} \mathrm{q}_{\mathrm{i}_{\mathrm{k}-1}}^{\mathrm{a}-1}$

$\mathrm{O}$ vetor da carga hidráulica que será avaliado iterativamente por:

$\mathrm{h}_{\mathrm{k}+1}=\mathrm{h}_{\mathrm{k}}-6 \mathrm{~A}^{\mathrm{T}} \mathrm{CA} \mathrm{C}^{-1} *$
$* \mathrm{Q}+\mathrm{A}^{\mathrm{T}} \mathrm{C}\left(\mathrm{Ah}_{\mathrm{k}}+\mathrm{A}_{\mathrm{r}} \mathrm{h}_{\mathrm{r}}\right) \mathrm{F}$

\section{Método Híbrido (MH)}

Os métodos MNR e MTL apresentam vantagens para se trabalhar com determinados tipos de problemas. De um modo geral, o MNR converge mais rápido (quadraticamente) quando comparado com o MTL, porém necessita de boas estimativas iniciais das cargas nos nós para que isto ocorra. Caso a aproximação inicial não esteja nas proximidades da solução final, o MNR pode consumir mais iteraçōes para convergir, podendo até não fazê-lo (Nielsen, 1989). Essa dificuldade é contornada implementando-se sub-rotinas para a determinação de melhores valores da solução inicial (Souza, 1994).

O MTL apresenta uma taxa de convergência inicial melhor do que o MNR, não precisando de valores iniciais tão bons. No entanto, o MTL tende a oscilar em torno da solução final, apresentando dificuldades de convergência para sistemas complexos (Hansen, Madsen e B, 1991).

O método proposto por Nielsen (1989), doravante chamado de híbrido $(\mathrm{MH})$, faz inicialmente algumas iterações, com o MTL, obtendo-se uma melhor aproximação das vazões e cargas hidráulicas nos nós. A aproximação assim obtida, será a solução inicial do MNR, que tende a convergir em um número menor de iteraçōes. Assim, o MTL serviria como uma rotina para determinação dos valores de partida para o método de Newton-Raphson propriamente dito.

Como as formulaçôes do MTL e MNR são semelhantes (3) e (6), a implementação do método Híbrido é realizada de acordo com a expressão (7): $\mathrm{h}_{\mathrm{k}+1}=\mathrm{h}_{\mathrm{k}}-\mathrm{c} 6 \mathrm{~A}^{\mathrm{T}} \mathrm{CA} \mathrm{e}^{-1}$

$\mathrm{Q}+\mathrm{A}^{\mathrm{T}} \mathrm{C}\left(\mathrm{Ah}_{\mathrm{k}}+\mathrm{A}_{\mathrm{r}} \mathrm{h}_{\mathrm{r}}\right) F$

em que $\mathrm{Y}=1$ quando $k \leq i i \mathrm{e}$ $\mathrm{\gamma}=\mathrm{a}$ quando $k>i i$, onde $i i$ é o número de iteraçôes iniciais com o MTL.

\section{Método Gradiente (MG)}

As equações de conservação de energia podem ser escritas matricialmente da seguinte forma (Todini e Pilati, 1987):

$\mathrm{Cq}+\mathrm{Ah}=-\mathrm{A}_{\mathrm{r}} \mathrm{h}_{\mathrm{r}}$

enquanto que a conservação de massa é expressa por:

$\mathrm{A}^{\mathrm{T}} \mathrm{q}=\mathrm{Q}$

O sistema de equações resultante é dado por:

$=\underset{\mathrm{A}^{\mathrm{T}}}{\mathrm{C}} \quad \mathrm{A}_{\mathrm{G}}={ }_{\mathrm{h}}^{\mathrm{q}} \mathrm{G}=={ }_{\mathrm{Q}}^{\mathrm{A}_{\mathrm{r}} \mathrm{h}_{\mathrm{r}} \mathrm{C}}$

Este sistema (10) é não linear, podendo ser resolvido através do esquema Newton-Raphson, para isso, é necessário efetuar uma diferenciação do sistema com relação à q e h, que resulta em:

$\stackrel{\text { a } C}{=} \mathrm{A}^{\mathrm{T}} \quad 0 \quad \mathrm{~A}_{\mathrm{G}}=\mathrm{dh}_{\mathrm{dh}}=\underset{\mathrm{dQ}}{=} \mathrm{dE}$

Após alguns procedimentos algébricos aplicados à expressão (11), encontramse as seguintes equações:

$\mathrm{q}_{\mathrm{k}+1}=\left(1-\mathrm{a}^{-1}\right) \mathrm{q}_{\mathrm{k}}-$

$-a^{-1} C^{-1} 7 A_{r} h_{r}+\mathrm{Ah}_{k+1} F$

$\left.\mathrm{h}_{\mathrm{k}+1}=-\left(\mathrm{A}^{\mathrm{T}} \mathrm{a}^{-1} \mathrm{C}^{-1} \mathrm{~A}\right)^{-1}\right)$

) $\mathrm{Ta}^{-1} \mathrm{~A}^{\mathrm{T}}\left(\mathrm{q}_{\mathrm{k}}+\mathrm{C}^{-1} \mathrm{~A}_{\mathrm{r}} \mathrm{h}_{\mathrm{r}}\right)+\left(\mathrm{Q}-\mathrm{A}^{\mathrm{T}} \mathrm{q}_{\mathrm{k}}\right) F$

\section{MODIFICACÃO DOS MÉTODOOS DE RESOLUÇÃO}

A inclusão dos eventuais vazamentos na rede e da utilização da demanda como uma variável dependente da pressão nos nós fará com que o vetor demanda (Q) seja função também da altura hidráulica (h). Desta forma, é preciso reformular o sistema de equaçôes original para atender a essa nova situação. As modificações no método de análise hidráulica são apresentadas a seguir.

A formulação empregada para vazamento neste trabalho foi proposta por Tucciarelli, Criminisi et al (1999), em que os coeficientes de perdas são considerados constantes por unidade de superfície dos tubos pertencentes a determinadas áreas da rede. Essa suposição é importante no processo de calibração 
de redes, em que se procura trabalhar com o menor número de parâmetros possível, além de ser melhor ajustada à formulação hidráulica de nós, que é empregada neste trabalho. Os autores consideraram que o vazamento pode ser expresso por:

$\operatorname{Vaz}_{j}=\left(h_{j}-z_{j}\right)^{i_{j}} !_{i=1}^{M_{j}}\left\{\frac{r}{2 i} d_{j i} L_{j i}\right.$

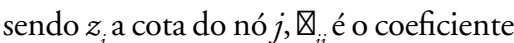
de perdas por unidade de superfície do tubo entre os nós $i$ e $j$; $\theta$ é o expoente de vazamento tomado como sendo 1,18 (Jowitt e Xu, 1990), e $d_{i j}$ é o diâmetro da tubulação.

A expressão empregada para DDP, conhecida como parabólica, foi proposta por Wagner, Shamir e Marks (1988) de acordo com o seguinte equacionamento:

$$
\begin{aligned}
& \mathrm{Q}_{\mathrm{j}}^{\text {tea }}=0 \quad \Rightarrow \mathrm{h}_{\mathrm{i}} \leq \mathrm{h}_{\mathrm{i}}^{\min }
\end{aligned}
$$

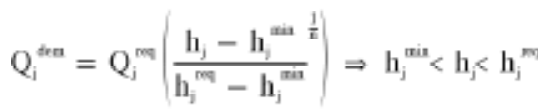

$$
\begin{aligned}
& \mathrm{Q}_{\mathrm{i}}^{\text {tex }}=\mathrm{Q}^{\mathrm{min}} \quad \Rightarrow \mathrm{h}_{\mathrm{i}} \geq \mathrm{h}_{\mathrm{i}}^{\text {m }}
\end{aligned}
$$

em que $Q^{\text {dem }}$ é a demanda atendida; $\mathrm{Q}^{\mathrm{req}}$ é a demanda potencial; $\mathrm{h}^{\text {min }}$ é a carga hidráulica mínima necessária haja suprimento de água, ou seja a cota mínima em que há consumo; $h^{\text {req }}$ é a carga hidráulica requerida para que a demanda seja completamente suprida e $n$ é o expoente tomado como 2 .

A adoção da vazão como sendo dependente da carga no nó torna necessário introduzir uma mudança na formulação original dos métodos para a análise hidráulica da rede. Nos métodos MNR, MTL E MH esta mudança ocorrerá na definiçãao da matriz Jacobiana que será:

$\mathrm{J}_{\mathrm{k}}=\mathrm{A}^{\mathrm{T}} \mathrm{CA}+\mathrm{C}_{\mathrm{vaz}}+\mathrm{C}_{\mathrm{Dem}}$

em que CVaz será avaliado pela formulação de Tucciarelli, Criminisi et al, 1999:

$\mathrm{C}_{\mathrm{Vaz}}=\operatorname{diag} \vec{\lambda}_{1}\left\{{ }_{1}\left(\mathrm{~h}_{1}-\mathrm{z}_{1}\right)^{\mathrm{i}_{1}-1}\right.$,

$\mathrm{i}_{2}\left\{{ }_{2}\left(\mathrm{~h}_{2}-\mathrm{z}_{2}\right)^{\mathrm{i}_{2}-1}, \ldots, \mathrm{i}_{\mathrm{NN}}\left\{\mathrm{in}_{\mathrm{NN}}\left(\mathrm{h}_{\mathrm{NN}}-\mathrm{z}_{\mathrm{NN}}\right)^{\mathrm{i}_{\mathrm{NN}}-1} \mathrm{~F}\right.\right.$

Esta expressão representa a derivada de $1^{\text {a }}$ ordem do termo de vazamento com relação à carga hidráulica $h_{j}$ de (14).

A matriz $\mathrm{C}_{\text {Dem }}$, que representa a derivada da equação de demanda dependente da pressão, é expressa por:

$C^{\prime \prime}=\operatorname{diag} 7 c_{1}, c_{2}, \ldots, c_{n} A$

em que:

$$
\begin{array}{ll}
c_{j}=\frac{1}{n_{j}} Q_{j}^{\text {req }} \frac{h_{j}-h_{j}^{\text {min }}}{h_{j}^{\text {des }}-h_{j}^{\frac{1}{n_{j}}-1}} \rho^{\text {min }} & \text { se } h_{j}^{\text {min }} \leq h_{j} \leq h_{j}^{\text {des }} \\
c_{j}=0 & \text { se } h_{j}^{\text {min }}>h_{j}>h_{j}^{\text {des }}
\end{array}
$$

Salgado, Todini e O’Connell (1993) propuseram uma modificação no MG de modo a considerar um componente da demanda como sendo uma função da carga hidráulica no nó.

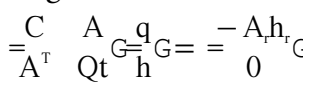

em que:

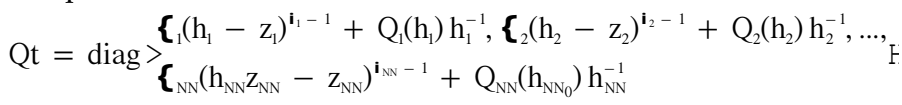

Desenvolvendo de modo semelhante ao feito no MG, obtêm-se as seguintes expressōes:

$$
\begin{aligned}
& \mathrm{q}_{\mathrm{k}+1}=\left(1-\mathrm{a}^{-1}\right) \mathrm{q}_{\mathrm{k}}- \\
& -\mathrm{a}^{-1} \mathrm{C}^{-1} 7 \mathrm{~A}_{\mathrm{r}} \mathrm{h}_{\mathrm{r}}+\mathrm{Ah}_{\mathrm{k}+1^{F}} \\
& \mathrm{~h}_{\mathrm{k}+1}=-\left(\mathrm{A}^{\mathrm{T}} \mathrm{a}^{-1} \mathrm{C}^{-1} \mathrm{~A}-\mathrm{R}\right)^{-1} \\
& \mathrm{a}^{-1} \mathrm{~A}^{\mathrm{T}}\left(\mathrm{q}_{\mathrm{k}}+\mathrm{C}^{-1} \mathrm{~A}_{\mathrm{r}} \mathrm{h}_{\mathrm{r}}\right)+ \\
& +\left(\mathrm{CH}_{\mathrm{r}}+\mathrm{Rh}_{\mathrm{r}}-\mathrm{A}^{\mathrm{T}} \mathrm{q}_{\mathrm{K}}\right) \mathcal{P} \\
& \text { em que: } \\
& \mathrm{R}=\mathrm{T}_{\mathrm{vaz}}+\mathrm{C}_{\mathrm{Dem}} \mathcal{F}
\end{aligned}
$$

\section{Válvulas}

Neste trabalho será avaliada uma rede com diferentes configuraçôes. Estas configuraçōes dizem respeito à consideração dos vazamentos, DDP e válvulas. As válvulas empregadas neste estudo são: válvula de retenção (VR) e válvula redutora de pressão, que têm o seu equacionamento apresentado a seguir.

Válvula de Retenção (VR): têm por finalidade fazer com que a água flua em apenas uma direção, fechando-se quando o fluxo começa a reverter. Seja um trecho $i$, com resistência igual a $K_{i}$, que possui uma válvula de retenção instalada. Esse dispositivo hidráulico pode operar de duas formas diferentes: a) fechada - se a altura de jusante for maior que a de montante a válvula será ativada fazendo com que: $K_{i}=+\infty q_{i}=0$. b) aberta - a VR estará inativa permanecendo inalterada a resistência do trecho.

Válvula Redutora de Pressão (VRP): servem para limitar a pressão à jusante, mantendo-a abaixo de valores que podem ser prejudiciais à rede. A VRP pode estar em três situaçóes: a) inativa - quando a carga a montante da válvula for inferior à pressão de serviço, nesta situação as condições do sistema permanecem inalteradas; b) fechada - quando a carga de jusante é maior que a de montante, neste caso a VRP se comportará como se fosse uma VR atuando uma resistência infinita c) ativa - em que permanece parcialmente fechada reduzindo a pressão à jusante da válvula, neste caso a resistência do trecho será:

$\mathrm{K}_{\mathrm{i}}^{\prime}=\mathrm{k}_{\mathrm{i}}+\mathrm{k}_{\mathrm{i}}^{\text {valv }}$

em que $K^{\text {valv }}$ é a resistência da válvula expressa por:

$\mathrm{K}_{\mathrm{i}}^{\text {valv }}=\frac{\mathrm{h}_{\text {valv }}-\mathrm{h}_{\mathrm{red}}}{\mathrm{q}_{\mathrm{i}}^{\mathrm{a}}}$

\section{Termo de relaxação para equação de demanda dirigida pela pressão}

Uma das dificuldades apontadas quando se trabalha com DDP diz respeito à descontinuidade da formulação (15), o que tem inviabilizado a adoção do equacionamento explícito para o método. Para exemplificar isto, considere um sistema adutor simples conforme a Figura 1. Para a formulação original proposta (15) tem-se que na primeira iteração, a carga hidráulica no nó será menor do que a mínima requerida ( $\mathrm{Fi}$ gura 1a). Assim na iteração seguinte, a demanda no nó será nula, e a pressão será a mesma do reservatório (Figura 1b). Este processo se repetirá indefinidamente fazendo a rotina entrar em um "loop infinito", impedindo assim a convergência para uma solução.

Se em uma situação relativamente simples, esta ocorrência implica na falta de convergência do método, para redes maiores, que contenham dispositivos como válvulas e bombas, tal fato pode inviabilizar completamente este tipo de abordagem. Assim, para se superar tal empecilho, este trabalho propõe uma relaxação no cálculo da DDP de modo a permitir a convergência, em que a demanda na iteração i seria calculada de seguinte forma: 
$Q^{i}=\frac{Q_{\text {calc }}^{\mathrm{i}}+\mathrm{Q}^{\mathrm{i}-1}}{2}$

Esta equação seria válida até que o método estabilizasse, pequeno erro entre duas iterações (neste trabalho empregou-se 0,1 ), a partir do qual voltaria a ser empregada a formulação original até atingir o critério de convergência.

\section{REDE EMPREGADA NO ESTUDO}

Para avaliar a eficiência e o comportamento dos modelos hidráulicos abordados neste trabalho foi escolhida uma rede exemplo usada por Souza (1994), que tem o seu layout apresentado na Figura 2. Esta rede é formada por 27 trechos conectados por 21 nós de consumo e 1 reservatório. A principal característica dessa configuração está no fato dela possuir os componentes hidráulicos que serão também avaliados neste trabalho: válvulas de retenção e redutora de pressão, sendo, ideal para a avaliação completa do comportamento de modelos de análise hidráulica. Os dados referentes aos nós da rede - cota, demanda, cargas hidráulicas desejada e mínima e coeficiente de vazamento - são apresentados na Tabela 1 , enquanto que as informações dos trechos - comprimento, diâmetros e coeficiente linear de vazamento - são encontradas na Tabela 2.

O foco das simulações efetuadas neste trabalho é verificar o funcionamento das metodologias de análise hidráulica da rede considerando a demanda dependente da pressão e os
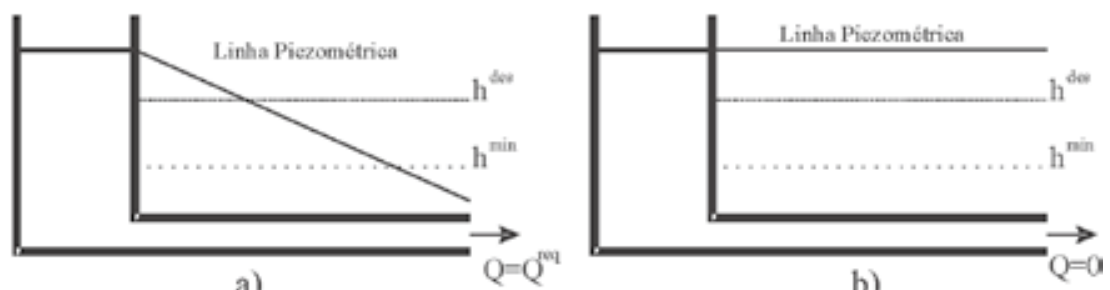

b)

Figura I - Comportamento da demanda dirigida pela pressão

vazamentos da rede. Por este motivo, foram feitas pequenas modificações nos dados originais. Quanto aos componentes da rede, a válvula redutora de pressão (VRP) funcionará quando a pressão à montante for superior a 90 e $25 \mathrm{mH}_{2} \mathrm{O}$ nas simulaçôes com e sem DDP respectivamente. A rugosidade dos tubos foi tomada como sendo igual a $1 \mathrm{~mm}$, uma vez que a equação empregada será a de Darcy-Weissbach para perda de carga, implicando em um valor de 2 para o expoente a de (3). Foram adotados dois níveis de água nos reservatórios, $50 \mathrm{~m}$ nas simulações em que a DDP foi empregada e $100 \mathrm{~m}$ nas restantes. $\mathrm{O}$ critério de convergência escolhido foi o de que a maior diferença entre as cargas hidráulicas nos nós obtidas em de duas iteraçôes consecutivas, fosse menor que uma tolerância $(\tau)$ préestabelecida, neste trabalho utilizou-se $\tau=10^{-5}$. A formulação apresentada para os métodos descritos neste trabalho foi implementada em linguagem de programação $\mathrm{C}++$, utilizando o compilador Borland C++ Builder 6 .

Os métodos de balanço hidráulico de redes foram testados para um total de 16 configuraçôes de componentes e diferentes comportamentos da rede quanto à demanda dependente da pressão e vazamentos (Tabela 3 )

\section{RESULTADOS}

Os métodos comparados neste trabalho apresentam formulações semelhantes, sendo diferentes em poucos detalhes. Do ponto de vista de eficiência computacional, o tempo gasto por esses métodos a cada iteração é muito similar, uma vez que os sistemas de equaçóes lineares, que consomem maior tempo computacional, resolvidos em cada passo são do mesmo tamanho $(n \times n)$ e possuem as mesmas características matriciais. Dessa forma, aferiu-se a eficiência das metodologias para análise hidráulica de rede a partir do número de iterações necessárias para que o método convergisse, considerando a tolerância pré-definida.

Os números de iterações necessários para a convergência dos métodos para cada configuração são mostrados na Tabela 4. Os valores das cargas hidráulicas nos nós e das vazôes nos trechos são mostrados nas Tabelas 5 e 6.

Tabela I - Dados referentes aos nós da rede empregada no estudo

\begin{tabular}{cccccccccccc}
\hline Nó & $\begin{array}{c}\mathrm{Z} \\
(\mathrm{m})\end{array}$ & $\begin{array}{c}\mathrm{Q} \\
(\mathrm{l} / \mathrm{s})\end{array}$ & $\begin{array}{c}\mathrm{h}^{\text {req }} \\
(\mathrm{m})\end{array}$ & $\begin{array}{c}\mathrm{h}^{\text {min }} \\
(\mathrm{m})\end{array}$ & $\mathrm{q}$ & Nó & $\begin{array}{c}\mathrm{Z} \\
(\mathrm{m})\end{array}$ & $\begin{array}{c}\mathrm{Q} \\
(\mathrm{l} / \mathrm{s})\end{array}$ & $\begin{array}{c}\mathrm{h}^{\text {req }} \\
(\mathrm{m})\end{array}$ & $\begin{array}{c}\mathrm{h}^{\text {min }} \\
(\mathrm{m})\end{array}$ & $\mathrm{q}$ \\
\hline 1 & 0 & 39 & 25 & 15 & 1.18 & 12 & 0 & 57 & 25 & 15 & 1.18 \\
2 & 0 & 57 & 25 & 15 & 1.18 & 13 & 0 & 18 & 25 & 15 & 1.18 \\
3 & 0 & 96 & 25 & 15 & 1.18 & 14 & 0 & 39 & 25 & 15 & 1.18 \\
4 & 0 & 18 & 25 & 15 & 1.18 & 15 & 0 & 39 & 25 & 15 & 1.18 \\
5 & 0 & 39 & 25 & 15 & 1.18 & 16 & 0 & 18 & 25 & 15 & 1.18 \\
6 & 0 & 57 & 25 & 15 & 1.18 & 17 & 0 & 39 & 25 & 15 & 1.18 \\
7 & 0 & 39 & 25 & 15 & 1.18 & 18 & 0 & 57 & 25 & 15 & 1.18 \\
8 & 0 & 39 & 25 & 15 & 1.18 & 19 & 0 & 39 & 25 & 15 & 1.18 \\
9 & 0 & 39 & 25 & 15 & 1.18 & 20 & 0 & 39 & 25 & 15 & 1.18 \\
10 & 0 & 39 & 25 & 15 & 1.18 & 21 & 0 & 18 & 25 & 15 & 1.18 \\
11 & 0 & 18 & 25 & 15 & 1.18 & 22 & $100 / 50$ & - & - & - & - \\
\hline
\end{tabular}


Tabela 2 - Dados referentes aos trechos da rede

\begin{tabular}{cccccccccccc}
\hline Trecho & $\begin{array}{c}\text { Nó } \\
\text { Mont. }\end{array}$ & $\begin{array}{c}\text { Nó } \\
\text { Jus. }\end{array}$ & $\begin{array}{c}\text { Comp } \\
(\mathrm{m})\end{array}$ & $\begin{array}{c}\text { Diam } \\
(\mathrm{mm})\end{array}$ & $\mathrm{j}$ & Trecho & $\begin{array}{c}\text { Nó } \\
\text { Mont. }\end{array}$ & $\begin{array}{c}\text { Nó } \\
\text { Jus. }\end{array}$ & $\begin{array}{c}\text { Comp } \\
(\mathrm{m})\end{array}$ & $\begin{array}{c}\text { Diam } \\
(\mathrm{mm})\end{array}$ & $\mathrm{j}$ \\
\hline 1 & 1 & 2 & 335 & 150 & $1 \mathrm{E}-07$ & 15 & 12 & 13 & 335 & 300 & $1 \mathrm{E}-07$ \\
2 & 3 & 2 & 335 & 150 & $1 \mathrm{E}-07$ & 16 & 12 & 10 & 244 & 300 & $1 \mathrm{E}-07$ \\
3 & 7 & 3 & 150 & 150 & $1 \mathrm{E}-07$ & 17 & 15 & 9 & 366 & 300 & $1 \mathrm{E}-07$ \\
4 & 5 & 1 & 244 & 200 & $1 \mathrm{E}-07$ & 18 & 18 & 12 & 305 & 450 & $1 \mathrm{E}-07$ \\
5 & 6 & 2 & 274 & 200 & $1 \mathrm{E}-07$ & 19 & 17 & 15 & 244 & 300 & $1 \mathrm{E}-07$ \\
6 & 11 & 7 & 305 & 200 & $1 \mathrm{E}-07$ & 20 & 14 & 15 & 305 & 150 & $1 \mathrm{E}-07$ \\
7 & 5 & 6 & 396 & 200 & $1 \mathrm{E}-07$ & 21 & 16 & 14 & 244 & 150 & $1 \mathrm{E}-07$ \\
8 & 4 & 5 & 183 & 150 & $1 \mathrm{E}-07$ & 22 & 17 & 16 & 305 & 150 & $1 \mathrm{E}-07$ \\
9 & 8 & 4 & 244 & 150 & $1 \mathrm{E}-07$ & 23 & 19 & 18 & 457 & 500 & $1 \mathrm{E}-07$ \\
10 & 9 & 8 & 244 & 250 & $1 \mathrm{E}-07$ & 24 & 21 & 19 & 305 & 500 & $1 \mathrm{E}-07$ \\
11 & 10 & 9 & 305 & 200 & $1 \mathrm{E}-07$ & 25 & 20 & 17 & 244 & 400 & $1 \mathrm{E}-07$ \\
12 & 10 & 6 & 183 & 300 & $1 \mathrm{E}-07$ & 26 & 22 & 20 & 457 & 400 & $1 \mathrm{E}-07$ \\
13 & 10 & 11 & 335 & 250 & $1 \mathrm{E}-07$ & 27 & 22 & 21 & 305 & 600 & $1 \mathrm{E}-07$ \\
14 & 13 & 11 & 274 & 300 & $1 \mathrm{E}-07$ & - & - & - & - & - & - \\
\hline
\end{tabular}

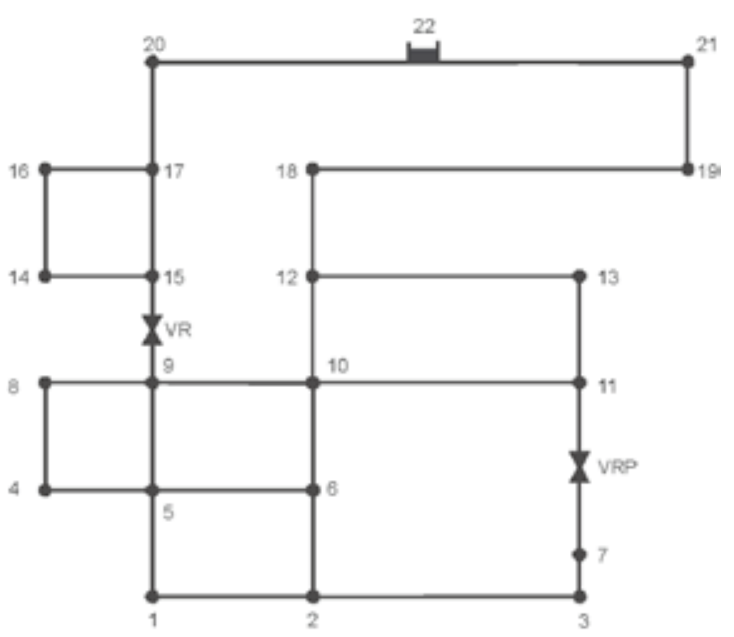

Figura 2 - Layout da Rede

Tabela 3 - Configurações da rede

\begin{tabular}{cccccccccc}
\hline Config & DDP & Vaz & VRP & VR & Config & DDP & Vaz & VRP & VR \\
\hline 1 & & & & & 9 & & $\mathrm{X}$ & & \\
2 & & & $\mathrm{X}$ & & 10 & & $\mathrm{X}$ & $\mathrm{X}$ & \\
3 & & & & $\mathrm{X}$ & 11 & & $\mathrm{X}$ & & $\mathrm{X}$ \\
4 & & & $\mathrm{X}$ & $\mathrm{X}$ & 12 & & $\mathrm{X}$ & $\mathrm{X}$ & $\mathrm{X}$ \\
5 & $\mathrm{X}$ & & & & 13 & $\mathrm{X}$ & $\mathrm{X}$ & & \\
6 & $\mathrm{X}$ & $\mathrm{X}$ & & 14 & $\mathrm{X}$ & $\mathrm{X}$ & $\mathrm{X}$ & \\
7 & $\mathrm{X}$ & & & $\mathrm{X}$ & 15 & $\mathrm{X}$ & $\mathrm{X}$ & & $\mathrm{X}$ \\
8 & $\mathrm{X}$ & $\mathrm{X}$ & $\mathrm{X}$ & 16 & $\mathrm{X}$ & $\mathrm{X}$ & $\mathrm{X}$ & $\mathrm{X}$ \\
\hline
\end{tabular}


Tabela 4 - Número de iterações dos métodos para diferentes configurações da rede

\begin{tabular}{|c|c|c|c|c|c|c|c|c|}
\hline \multirow[t]{2}{*}{ Simulação } & \multicolumn{4}{|c|}{ Com relaxação } & \multicolumn{4}{|c|}{ Sem relaxação } \\
\hline & Nielsen & Teoria linear & NR & Gradiente & Nielsen & Teoria linear & NR & Gradiente \\
\hline 1 & 7 & 22 & 6 & 5 & 7 & 22 & 6 & 5 \\
\hline 2 & 20 & 31 & 19 & 17 & 17 & 31 & 16 & 15 \\
\hline 3 & 13 & 23 & 27 & 21 & 13 & 23 & 27 & 21 \\
\hline 4 & 18 & 30 & $\mathrm{NC}$ & 21 & 18 & 30 & $\mathrm{NC}$ & 21 \\
\hline 5 & 14 & 31 & 20 & 11 & 15 & 20 & $\mathrm{NC}$ & $\mathrm{NC}$ \\
\hline 6 & 26 & 20 & $\mathrm{NC}$ & $\mathrm{NC}$ & 32 & 21 & $\mathrm{NC}$ & $\mathrm{NC}$ \\
\hline 7 & 15 & 19 & $\mathrm{NC}$ & 20 & 15 & 19 & $\mathrm{NC}$ & 26 \\
\hline 8 & 27 & 23 & $\mathrm{NC}$ & $\mathrm{NC}$ & 44 & 24 & $\mathrm{NC}$ & $\mathrm{NC}$ \\
\hline 9 & 8 & 22 & 7 & 6 & 8 & 22 & 7 & 6 \\
\hline 10 & 19 & 29 & 19 & 17 & 17 & 30 & 17 & 16 \\
\hline 11 & 8 & 22 & 17 & 21 & 8 & 22 & 17 & 21 \\
\hline 12 & 16 & 27 & $\mathrm{NC}$ & 21 & 14 & 25 & $\mathrm{NC}$ & 21 \\
\hline 13 & 16 & 20 & 29 & 10 & 16 & 20 & $\mathrm{NC}$ & $\mathrm{NC}$ \\
\hline 14 & 40 & 35 & $\mathrm{NC}$ & $\mathrm{NC}$ & 42 & 38 & $\mathrm{NC}$ & $\mathrm{NC}$ \\
\hline 15 & 18 & 19 & 46 & 20 & 19 & 20 & $\mathrm{NC}$ & $\mathrm{NC}$ \\
\hline 16 & 32 & 29 & $\mathrm{NC}$ & $\mathrm{NC}$ & 38 & 29 & $\mathrm{NC}$ & $\mathrm{NC}$ \\
\hline
\end{tabular}

Tabela 5 - Cargas hidráulicas nos nós $\left(\mathrm{em}_{\mathrm{mH}} \mathrm{O}\right)$ para as diferentes configurações da Rede

\begin{tabular}{|c|c|c|c|c|c|c|c|c|c|c|c|c|c|c|c|c|}
\hline \multirow[t]{2}{*}{ Nó } & \multicolumn{16}{|c|}{ Simulação } \\
\hline & 1 & 2 & 3 & 4 & 5 & 6 & 7 & 8 & 9 & 10 & 11 & 12 & 13 & 14 & 15 & 16 \\
\hline 1 & 58.8 & 56.8 & 44.3 & 43.4 & 21.0 & 21.0 & 19.2 & 19.4 & 52.5 & 51.2 & 37.9 & 37.7 & 20.4 & 20.7 & 18.8 & 22.8 \\
\hline 2 & 58.5 & 55.9 & 44.5 & 43.4 & 21.0 & 21.1 & 19.5 & 19.7 & 52.4 & 50.6 & 38.2 & 38.0 & 20.5 & 20.7 & 19.2 & 23.6 \\
\hline 3 & 47.5 & 36.4 & 35.2 & 31.2 & 18.2 & 17.9 & 176 & 16.7 & 41.5 & 34.1 & 29.0 & 28.2 & 18.0 & 16.9 & 17.4 & 28.6 \\
\hline 4 & 63.1 & 62.0 & 45.9 & 45.2 & 22.7 & 22.8 & 19.6 & 19.9 & 57.1 & 56.4 & 39.5 & 39.3 & 22.0 & 22.4 & 19.3 & 22.6 \\
\hline 5 & 61.3 & 59.9 & 45.9 & 45.2 & 22.0 & 22.1 & 19.7 & 20.0 & 55.2 & 54.3 & 39.5 & 39.4 & 21.4 & 21.8 & 19.3 & 22.7 \\
\hline 6 & 69.8 & 69.2 & 57.5 & 57.2 & 27.1 & 27.4 & 24.7 & 25.5 & 64.5 & 64.1 & 51.9 & 51.9 & 26.3 & 27.2 & 24.2 & 27.6 \\
\hline 7 & 59.9 & 44.2 & 48.7 & 42.7 & 22.9 & 21.7 & 21.8 & 18.4 & 54.3 & 43.4 & 43.0 & 41.7 & 22.4 & 18.4 & 21.4 & 29.3 \\
\hline 8 & 71.5 & 71.2 & 47.7 & 47.2 & 27.1 & 27.3 & 20.1 & 20.5 & 66.3 & 66.1 & 41.2 & 41 & 26.1 & 26.8 & 19.7 & 22.6 \\
\hline 9 & 73.9 & 73.7 & 49.0 & 48.5 & 28.9 & 29.1 & 20.7 & 21.1 & 68.9 & 68.8 & 42.6 & 42.5 & 27.9 & 28.6 & 20.3 & 23.1 \\
\hline 10 & 74.8 & 74.7 & 63.4 & 63.3 & 30.5 & 30.8 & 27.9 & 28.9 & 70.0 & 70.0 & 58.2 & 58.2 & 29.6 & 30.7 & 27.3 & 30.5 \\
\hline 11 & 75.1 & 75.4 & 65.0 & 65.1 & 31.0 & 31.4 & 28.8 & 30.5 & 70.4 & 70.6 & 59.9 & 60.0 & 30.1 & 31.9 & 28.2 & 32.4 \\
\hline 12 & 83.9 & 84.0 & 77.7 & 77.7 & 37.3 & 37.5 & 35.9 & 36.6 & 80.5 & 80.6 & 74.1 & 74.1 & 36.6 & 37.4 & 35.3 & 37.4 \\
\hline 13 & 78.6 & 78.8 & 70.1 & 70.2 & 33.4 & 33.7 & 31.5 & 32.8 & 74.3 & 74.4 & 65.5 & 65.5 & 32.5 & 33.9 & 30.9 & 34.2 \\
\hline 14 & 74.4 & 74.3 & 86.6 & 86.6 & 27.3 & 27.4 & 36.6 & 36.6 & 69.6 & 69.6 & 83.4 & 83.4 & 26.1 & 26.5 & 35.4 & 35.4 \\
\hline 15 & 79.1 & 79.0 & 92.5 & 92.5 & 32.3 & 32.5 & 42.5 & 42.5 & 74.9 & 74.9 & 90.3 & 90.3 & 31.3 & 31.8 & 41.7 & 41.7 \\
\hline 16 & 75.6 & 75.5 & 87.3 & 87.3 & 28.3 & 28.4 & 37.3 & 37.3 & 70.9 & 70.8 & 84.1 & 84.1 & 27.1 & 27.6 & 36.1 & 36.1 \\
\hline 17 & 82.9 & 82.9 & 93.3 & 93.3 & 35.3 & 35.4 & 43.3 & 43.3 & 79.4 & 79.4 & 91.3 & 91.3 & 34.5 & 34.8 & 42.5 & 42.5 \\
\hline 18 & 88.8 & 88.9 & 84.7 & 84.7 & 41.1 & 41.2 & 40.1 & 40.6 & 86.3 & 86.3 & 82.0 & 82.0 & 40.5 & 41.0 & 39.6 & 41.0 \\
\hline 19 & 94.2 & 94.2 & 92.1 & 92.1 & 45.3 & 45.4 & 44.8 & 45.1 & 92.7 & 92.7 & 90.6 & 90.6 & 44.9 & 45.2 & 44.5 & 45.2 \\
\hline 20 & 90.9 & 90.8 & 96.1 & 96.1 & 42.1 & 42.1 & 46.1 & 46.1 & 88.9 & 88.8 & 94.9 & 94.9 & 41.5 & 41.7 & 45.6 & 45.6 \\
\hline 21 & 98.3 & 98.3 & 97.7 & 97.7 & 48.6 & 48.6 & 48.5 & 48.5 & 97.8 & 97.8 & 97.2 & 97.2 & 48.5 & 48.6 & 48.4 & 48.6 \\
\hline Reserv. & 100 & 100 & 100 & 100 & 50 & 50 & 50 & 50 & 100 & 100 & 100 & 100 & 50 & 50 & 50 & 50 \\
\hline
\end{tabular}


Tabela 6 - Vazões nos trechos (em I/s) para as diferentes configurações da rede

\begin{tabular}{|c|c|c|c|c|c|c|c|c|c|c|c|c|c|c|c|c|}
\hline \multirow[t]{2}{*}{ Trecho } & \multicolumn{16}{|c|}{ Simulação } \\
\hline & 1 & 2 & 3 & 4 & 5 & 6 & 7 & 8 & 9 & 10 & 11 & 12 & 13 & 14 & 15 & 16 \\
\hline 1 & 5.3 & 10.2 & -4.3 & -2.0 & -2.2 & -1.8 & -6.3 & -5.4 & 4.5 & 8.0 & -5.5 & -5.0 & -2.5 & -1.3 & -6.3 & -6.3 \\
\hline 2 & -37.0 & -49.5 & -34.0 & -39.1 & -18.4 & -19.6 & -15.1 & -18.8 & -36.8 & -45.6 & -33.7 & -34.8 & -17.5 & -21.6 & -14.5 & -14.5 \\
\hline 3 & 59.0 & 46.5 & 62.0 & 56.9 & 36.1 & 32.3 & 34.0 & 21.3 & 60.1 & 51.1 & 62.9 & 61.8 & 35.1 & 20.5 & 33.2 & 33.2 \\
\hline 4 & 44.3 & 49.2 & 34.7 & 37.0 & 28.0 & 28.5 & 19.0 & 20.5 & 45.2 & 48.6 & 34.7 & 35.1 & 26.8 & 28.7 & 18.4 & 18.4 \\
\hline 5 & 88.7 & 96.3 & 95.3 & 98.1 & 64.8 & 65.8 & 59.8 & 63.2 & 91.9 & 97.1 & 98.0 & 98.6 & 63.2 & 66.9 & 58.7 & 58.7 \\
\hline 6 & 98.0 & 85.5 & 101.0 & 95.9 & 70.8 & 64.2 & 66.1 & 44.1 & 100.6 & 91.3 & 103.0 & 101.9 & 69.2 & 43.7 & 64.9 & 64.9 \\
\hline 7 & -63.4 & -66.5 & -74.6 & -75.9 & -49.0 & -49.7 & -48.8 & -51.0 & -66.5 & -68.6 & -77.2 & -77.5 & -48.0 & -50.5 & -48.1 & -48.1 \\
\hline 8 & 19.9 & 21.7 & -0.9 & 0.2 & 11.7 & 11.8 & -3.2 & -3.0 & 20.5 & 21.7 & -1.7 & -1.5 & 11.0 & 11.4 & -3.3 & -3.3 \\
\hline 9 & 37.9 & 39.7 & 17.1 & 18.2 & 27.4 & 27.7 & 9.1 & 9.6 & 39.7 & 40.9 & 17.1 & 17.3 & 26.5 & 27.3 & 8.8 & 8.8 \\
\hline 10 & 76.9 & 78.7 & 56.1 & 57.2 & 66.4 & 66.7 & 37.0 & 38.6 & 80.8 & 82.1 & 57.3 & 57.5 & 66.2 & 67.0 & 36.2 & 36.2 \\
\hline 11 & -22.7 & -23.9 & -95.1 & -96.2 & -30.6 & -31.6 & -66.5 & -69.2 & -24.9 & -25.9 & -99.1 & -99.3 & -31.8 & -35.6 & -65.7 & -65.7 \\
\hline 12 & 209.1 & 219.8 & 226.9 & 230.9 & 170.8 & 172.5 & 164.8 & 171.2 & 219.4 & 226.7 & 235.3 & 236.2 & 169.5 & 175.8 & 162.7 & 162.7 \\
\hline 13 & -25.1 & -35.5 & -53.3 & -57.3 & -28.9 & -33.1 & -40.9 & -53.6 & -25.9 & -33.6 & -54.7 & -55.6 & -29.0 & -44.2 & -39.9 & -39.9 \\
\hline 14 & 141.1 & 139.0 & 172.3 & 171.2 & 117.7 & 115.2 & 125.0 & 115.7 & 149.9 & 148.2 & 180.2 & 179.9 & 118.1 & 108.0 & 124.6 & 124.6 \\
\hline 15 & 159.1 & 157.0 & 190.3 & 189.2 & 135.7 & 133.2 & 143.0 & 133.7 & 172.5 & 170.9 & 202.2 & 201.9 & 137.9 & 127.9 & 144.2 & 144.2 \\
\hline 16 & 245.7 & 247.2 & 307.7 & 308.8 & 211.5 & 210.0 & 229.5 & 225.8 & 263.8 & 264.5 & 323.9 & 324.1 & 213.7 & 208.7 & 229.7 & 229.7 \\
\hline 17 & 93.2 & 93.8 & 0.0 & 0.0 & 74.9 & 74.1 & 0.0 & 0.0 & 99.9 & 100.1 & 0.0 & 0.0 & 75.1 & 72.2 & 0.0 & 0.0 \\
\hline 18 & 461.8 & 461.2 & 555.0 & 555.0 & 404.2 & 400.3 & 429.5 & 416.5 & 502.0 & 501.1 & 591.0 & 590.9 & 412.0 & 397.0 & 434.2 & 434.2 \\
\hline 19 & 157.5 & 158.0 & 67.5 & 67.5 & 140.0 & 139.2 & 67.5 & 67.5 & 171.0 & 171.2 & 76.4 & 76.4 & 142.6 & 139.9 & 71.0 & 71.0 \\
\hline 20 & -25.3 & -25.2 & -28.5 & -28.5 & -26.1 & -26.1 & -28.5 & -28.5 & -26.7 & -26.7 & -30.7 & -30.7 & -26.6 & -26.8 & -29.3 & -29.3 \\
\hline 21 & 13.7 & 13.8 & 10.5 & 10.5 & 12.9 & 12.9 & 10.5 & 10.5 & 14.2 & 14.2 & 10.7 & 10.7 & 13.0 & 12.9 & 10.6 & 10.6 \\
\hline 22 & 31.7 & 31.8 & 28.5 & 28.5 & 30.9 & 30.9 & 28.5 & 28.5 & 34.2 & 34.2 & 31.1 & 31.1 & 31.6 & 31.5 & 29.5 & 29.5 \\
\hline 23 & 518.8 & 518.2 & 612.0 & 612.0 & 461.2 & 457.3 & 486.5 & 473.5 & 570.1 & 569.1 & 658.4 & 658.3 & 473.5 & 458.6 & 495.6 & 495.6 \\
\hline 24 & 557.8 & 557.2 & 651.0 & 651.0 & 500.2 & 496.3 & 525.5 & 512.5 & 621.6 & 620.7 & 709.6 & 709.5 & 517.9 & 503.0 & 539.9 & 539.9 \\
\hline 25 & 228.2 & 228.8 & 135.0 & 135.0 & 209.9 & 209.1 & 135.0 & 135.0 & 249.5 & 249.7 & 152.7 & 152.7 & 215.2 & 212.4 & 142.0 & 142.0 \\
\hline 26 & 267.2 & 267.8 & 174.0 & 174.0 & 248.9 & 248.1 & 174.0 & 174.0 & 295.8 & 296.0 & 199.6 & 199.6 & 257.2 & 254.4 & 184.3 & 184.3 \\
\hline 27 & 575.8 & 575.2 & 669.0 & 669.0 & 518.2 & 514.3 & 543.5 & 530.5 & 651.4 & 650.4 & 739.3 & 739.2 & 541.0 & 526.2 & 563.0 & 563.0 \\
\hline
\end{tabular}

A partir dos resultados apresentados na Tabela 4 verifica-se que o MG foi o mais eficiente para as configurações mais simples que não consideram a DDP e os vazamentos (Configurações 1 a 4), bem como nas situações em que a DDP e vazamentos foram consideradas sem a presença de componentes hidráulicos (Configuraçôes 5, 9 e 13). No entanto, para as outras redes o desempenho do método foi inferior ao $\mathrm{MH}$ e em alguns casos (Configuraçôes 6, 8, 14 e 16), não apresentou convergência. Verifica-se assim uma grande capacidade do MG para redes mais simples e uma dificuldade de encontrar soluções para sistemas mais complexos, onde ocorrem descontinuidades no sistema, geradas principalmente pela presença de VRPs.

Para configurações mais complexas (Configuraçóes 3, 4, 7, 11, 12, 13 e 15), o $\mathrm{MH}$ foi o que apresentou melhor convergência (Tabela 4). Isto decorre especialmente pela estabilidade numérica proporcionada pelo MTL, que é empregado nas primeiras iteraçoes daquele método. Embora o $\mathrm{MH}$ não tenha sido o método que convergiu em menos iteraçôes nas outras configuraçôes, este não ficou muito distante dos métodos mais rápidos, conseqüência da sua capacidade em lidar com sistemas simples ou complexos.

O MTL foi o mais lento na análise de configurações menos complexas, chagando a gastar 3 vezes mais tempo do que o MG (Configuraçóes 1, 5 e 9). Entretanto, o MTL mostrou-se o mais rápido nas situações mais complexas analisadas. Isto se deve principalmente à estabilidade do método que permite uma aproximação gradual, porém estável, da solução final e permite ao método lidar bem com as descontinuidades das funçōes resultante das configuraçôes mais complexas. No caso dos métodos mais rápidos, como o Gradiente e Newton-Raphson, pode-se incorrer em dificuldades tais como instabilidade numérica e não convergência. Mesmo que os equacionamentos do MNR (3) e do MTL (6) sejam muito semelhantes, o MTL convergiu para todas as situa- 
ções enquanto que o MNR apresentou dificuldade de convergência para os casos mais complexos.

A relaxação introduzida melhorou a convergência do MG nas Simulações 3 e 4 e permitiu a convergência do MG e do MNR para a situação de DDP (Simulação 5 e 13). No entanto, a relaxação da função DDP não alterou a convergência dos métodos MTL e MH. Isto se deve ao fato do MTL já ter incorporado em sua formulação o principio de cálculo das vazôes de cada interação levando-se em conta a vazão da iteração anterior.

Das configurações analisadas, aquelas que possuem válvulas redutoras de pressão foram as que requereram maior esforço computacional, fato motivado pelo tipo de condição de contorno que este componente apresenta, com três possíveis estados e descontinuidade nas derivadas de primeira ordem. As condições de contorno das válvulas de retenção, embora mais simples, são descontínuas, operando em apenas dois tipos de condições: aberta ou fechada, provocando também um aumento no número de iterações.

\section{CONCLUSÕES}

Os modelos de análise hidráulica de redes de distribuição são ferramentas essenciais nos estudos modernos de projeto e operação dos sistemas de abastecimento, uma vez que eles permitem avaliar diferentes cenários de funcionamento e auxiliam o processo de tomada de decisão. Para que o uso destes modelos seja ampliado é preciso que os mesmos sejam adaptados para descrever a situaçōes mais realísticas que os sistemas, principalmente os brasileiros, enfrentam atualmente, como pressōes insuficientes em alguns pontos da rede e vazamentos.

Esse trabalho procurou comparar as principais formulações de análise hidráulica de redes em regime permanente desenvolvidas ao longo das últimas décadas para situaçôes de demanda dirigida pela pressão (DDP) e vazamentos na rede. As formulações MNR (Mét. Newton-Raphson), MTL MTL (Mét.Teoria Linear), MH (Mét. Híbrido) e MG (Mét.Gradiente) foram modificadas de modo a considerar os vazamentos e a DDP dentro do modelo e implementadas em linguagem de programação $\mathrm{C}++$.
O MG mostrou ser o mais robusto, uma vez que trabalha diretamente com as duas variáveis envolvidas no problema, carga hidráulica e vazão. Isto se verifica pela sua melhor adaptação a configurações menos complexas, como nas situaçốes em que a DDP e os vazamentos não são considerados, ou quando estes ocorrem individualmente sem a presença de válvulas. Esta eficiência do MG o faz estar presente na maior parte dos softwares disponíveis no mercado. Ao se avaliar cenários mais complexos, o MG apresentou alguns problemas de convergência, havendo casos em que o mesmo não convergiu, mesmo após a incorporação da rotina de relaxação.

O MH mostrou-se o método que melhor se adapta aos diferentes cenários de complexidade, pois alia as características positivas do MTL, de estabilidade para as iteraçōes iniciais, e do MNR, relativas à convergência para a solução final. O MTL apresentou uma fraca eficiência para redes mais simples, sendo até três vezes mais lento que o $\mathrm{MG}$, porém em sistemas mais complexos, foi o método que melhor se comportou. O MNR revelou-se um algoritmo muito instável para analisar redes mais complexas, produzindo o pior resultado dentre as metodologias avaliadas, na maioria dos casos.

Para melhorar a eficiência dos métodos na análise de redes com DDP foi proposta uma forma de cálculo da vazão do sistema, considerando a estimativa da iteração anterior. Esta relaxação melhorou a taxa de convergência do método $\mathrm{MG}$ e MNR na maioria das configurações, porém, para o MTL e MH não houve melhora. Isto ocorreu porque a linearização do MTL emprega um esquema de atualização de vazôes semelhante à relaxação proposta neste trabalho.

\section{AGRADECIMENTOS}

Este trabalho teve apoio da FAPESP que subsidiou os estudos do primeiro autor por meio de bolsa de Doutorado, e do CNPq, Processo 478810/2006-7, cujo de auxílio financeiro possibilitou o prosseguimento das investigaçōes. Agradecemos também aos revisores que deram importantes contribuições à versão final deste artigo.

\section{REFERÊNCIAS}

ACKLEY, J. R. L., TANYIMBOH, T. T., TAHAR, B. Head Driven Analysis of Water
Distribution Systems. In: B. Ulanicki, B. Boulbeck, et al (Ed.). Water Software Systems: Theory and Applications. London: Research Studies Press, v.1, p.183-192, 2001.

COLLINS, M., COOPER, L., HELGASON, R. Solving the Pipe Network Analysis Problem Using Optimization Techniques. Management Science, v.24, n.7, p.747-760. 1978.

CROSS, H. Analysis of Flow in Networks of Conduits or Conductors. Enginnering Experimental Station: University of Illinois. (Bulletin No 286), 1936.

FORMIGA, K. T. M. Otimização multiobjetivo de projetos de redes de distribuição de água. (Tese de Doutorado). Hidráulica e Saneamento, Universidade de São Paulo, São Carlos, 276 p. 2005.

FUJIWARA, O. E GANESHARAJAH, T. Reliability assessment of water supply systems with storage and distributions networks. Water Resources Research, v.29, n.8, p.2917-2924. 1993.

GERMANOPOULOS, G. E JOWITT, P. W. Leakage reduction by excessive pressure minimization in a water supply network. Proceedings of the Institution of Civil Engineers, v.87, n.2, p.195-214. 1989.

GOMES, H. P. Sistema de Abastecimento de Água - Dimensionamento Econômico e Operação de Redes e Elevatórias. João Pessoa: Editora Universitária - UFPB. 242 p. 2004.

GUPTA, R. E BHAVE, P. R. Comparison of Methods for Predicting Deficient-Network Performance. Journal of Water Resources Planning and Management, ASCE, v.122, n.3, p.214-217. 1996.

HANSEN, C. T., MADSEN, K. E B, N. H. Optimization of pipe networks. Mathematical Programming, v.52, n.1, p.45-58. 1991.

ISAACS, L. T. E MILLS, K. G. Linear Theory Method for Pipe Network Analysis. Journal of Hydraulic Engineering, v.106, n.7, p.1191-2001. 1980.

JOWITT, P. W. E Xu, C. Optimal valve control in water-distribution networks. Journal of Water Resources Planning and Management, ASCE, v.116, n.4, p.455-472. 1990.

LEMIEUX, P. F. Efficient Algorithm for Distribution Networks. Journal of the Hydraulics Division, v.98, n.11, p.1911-1920. 1972.

MARTÍNEZ, F., CONEJOS, P. E VERCHER, J. Developing an Integrated Model for Water Distribution Systems Considering both Distributed Leakage and Pressure-Dependent Demands. 26th ASCE Water Resources Planning and Management Division Conference. Tempe, AZ: ASCE, 1999.

NIELSEN, H. B. Methods for Analyzing Pipe Networks. Journal of Hydraulic Engineering, ASCE, v.125, n.2, p.139-157 1989.

REDDY, L. E ELANGO, K. Analysis of Water Distribution Networks with Head Dependent Outlets. Civil Engineering Systems, v.6, n.3, p.102-110. 1989.

SALGADO, R., ROJO, J. E ZEPEDA, S. Extended gradient method for fully non-linear head and flow analysis in pipe networks. In: B. Coulbeck (Ed.). Integrated Computer Applications of Water Supply - Methods and Procedures for Systems Simulation and Control. New York: John Wiley \& Sons, Inc., v.1, p.49-60 1993. 
SHAMIR, U. E HOWARD, C. D. D. Water Distribution Systems Analysis. Journal of Hydraulics Division, ASCE, v.94, n.1, p.219234. 1968.

SOARES, A. K., CHEUNG, P. B., REIS, L. F. R. Avaliação das perdas fisicas de um setor da rede de abastecimento de água de Campo Grande-MS via modelo inverso. Revista Brasileira de Engenharia Sanitária e Ambiental, v.9, n.4 p.312-321. 2004

SOUZA, R. S. Aspectos computacionais da analise de redes de distribuição de água com componentes hidráulicos em regime permanente. (Dissertação de Mestrado). Hidráulica e Saneamento, Universidede de São Paulo, São Carlos, 236 p. 1994.

TODINI, E. A more realistic to the "extended period simulation" of water distribution networks. In: C. Maksimovic, D. Butler, et al (Ed.). Advances in Water Supply Management. Lisse A. A. Balkema Publishers, p.732, 2003.

TODINI, E. E PILATI, S. A Gradient Algorithm for ht Analysis of Pipe Networks. In: (Ed.) International Conference on Computer Applications for Water Supply and Distribution: Leicester Polytechnic, p.1-20, 1987.

TUCCIARELLI, T., CRIMINISI, A. E TERMINI, D. Leak Analysis in Pipeline Systems by Means of Optimal Valve Regulation. Journal of Hydraulic Engineering, ASCE, v.125, n.3, p.277-285. 1999.

WAGNER, J. M., SHAMIR, U. E MARKS, D. H. Water Distribution Reliability: Analytical Methods. Journal of Water Resources Planning and Management, ASCE, v.114, n.3, p.276-292. 1988

WOOD, D. J. E CHARLES, C. O. A. Hydraulic Network Analysis Using Linear Theory. Journal of Hydraulics Division, ASCE, v.98, n.7, p.1157-1170. 1972

Endereço para correspondência:

Klebber Teodomiro Martins

Formiga

Escola de Engenaria Civil

Universidade Federal de Goiás

Praça Universitária $s / n^{\circ}$

Setor Universitário

74605-220 Goiânia -GO - Brasil

E-mail: klebber.formiga@gmail.com 